\title{
Cuidados farmacológicos na interação medicamentosa: clonazepam com álcool
}

\author{
Pharmacological care in drug interaction: clonazepam with alcohol \\ Atención farmacológica en interacción farmacológica: clonazepam con alcohol
}

Recebido: 03/11/2021 | Revisado: 10/11/2021 | Aceito: 16/11/2021 | Publicado: 17/11/2021

\author{
Jessica Scarlett Pereira Jacaúna \\ ORCID: https://orcid.org/0000-0003-3357-6535 \\ Universidade Nilton Lins, Brasil \\ E-mail: jessicascarlett@ hotmail.com \\ Omero Martins Rodrigues Junior \\ ORCID: https://orcid.org/0000-0002-8552-3278 \\ Universidade Nilton Lins, Brasil \\ E-mail: omeromartins.farma@gmail.com
}

\begin{abstract}
Resumo
Os medicamentos são ferramentas importantes em saúde, porém a sua administração deve ser realizada com precaução, avaliando a relação benefício/risco e a eficácia para indicação clínica a que se destinam. Os efeitos do medicamento clonazepam quando associado ao uso de álcool é prejudicial à saúde, fazendo que altere a dose e potencializando seus efeitos. Quando acontece essa interação o indivíduo tende a sofrer as consequências, que geralmente são graves. Em virtude disso, o presente artigo busca salientar a interação medicamentosa do clonazepam e álcool, demonstrando como o uso dos dois juntos afeta o SNC (Sistema Nervoso Central). O artigo terá quatro partes, na primeira falaremos sobre o que é o clonazepam, na segunda parte mostrará o que acontece quando ingerido com o álcool, na terceira vamos apresentar uma discussão sobre o assunto e por fim os resultados/conclusões.
\end{abstract}

Palavras-chave: Interação medicamentosa; Clonazepam; Álcool; Riscos.

\begin{abstract}
Medications are important health tools, but their administration must be performed with caution, evaluating the benefit/risk ratio and effectiveness for the clinical indication for which they are intended. The effects of the drug clonazepam when associated with the use of alcohol is harmful to health, causing the dose to change and enhancing its effects. When this interaction happens, the individual tends to suffer the consequences, which are usually serious. As a result, this article seeks to highlight the drug interaction of clonazepam and alcohol, demonstrating how the use of both together affects the SNC (Central Nervous System). The article will have four parts, in the first part we will talk about what clonazepam is, in the second part we will show what happens when ingested with alcohol, in the third part we will present a discussion on the subject and finally the results/conclusions.
\end{abstract}

Keywords: Drug interaction; Clonazepam; Alcohol; Scratchs.

\section{Resumen}

Los medicamentos son herramientas sanitarias importantes, pero su administración debe llevarse a cabo con precaución, evaluando la relación beneficio/riesgo y la eficacia para la indicación clínica a la que están destinados. Los efectos de la droga clonazepam cuando se asocia con el consumo de alcohol es perjudicial para la salud, haciendo que cambie la dosis y potenciando sus efectos. Cuando ocurre esta interacción el individuo tiende a sufrir las consecuencias, que suelen ser graves. Como resultado, este artículo busca resaltar la interacción farmacológica del clonazepam y el alcohol, demostrando cómo el uso de los dos juntos afecta al SNC (Sistema Nervioso Central). El artículo tendrá cuatro partes, en la primera hablaremos sobre qué es el clonazepam, en la segunda parte mostraremos lo que sucede cuando se ingiere con alcohol, en la tercera presentaremos una discusión sobre el tema y finalmente los resultados/conclusiones.

Palabras clave: Interacción medicamentosa; Clonazepam; Alcohol; Riesgos.

\section{Introdução}

Por definição da Agência Nacional de Vigilância Sanitária (Anvisa), interação medicamentosa é a alteração dos efeitos farmacológicos entre dois ou mais medicamentos administrados concomitantemente, podendo resultar em um aumento ou diminuição na eficácia terapêutica ou nos eventos adversos causados por estes, ou ainda no aparecimento de novos efeitos. Portanto, podem resultar em interações benéficas ou prejudiciais (Lucho, 2016). 
A interação medicamentosa caracteriza-se como um evento onde os efeitos de um fármaco podem ser alterados pela presença de outro fármaco, alimento ou substâncias diversas (por exemplo: tabaco, plantas medicinais, álcool). Estudos mostram que as interações medicamentosas estão relacionadas a altos custos para o indivíduo e para os sistemas de saúde, além de aumentar o tempo de permanência em hospitais. As interações geralmente causam modificações na farmacocinética e/ou farmacodinâmica do(s) fármaco(s). Essas interações podem ser classificadas em farmacocinéticas, farmacodinâmicas, farmacêuticas ou de efeito (Piato, 2013).

As interações envolvendo medicamento e o álcool podem ser de vários tipos, de modo que, mesmo que essa ingestão seja de pequena ou grande quantidade ela poderá inibir ou induzir o mecanismo de ação de alguns fármacos (Gotardelo, 2015).

Um importante representante da classe dos benzodiazepínicos é o clonazepam, considerado fármaco potente e de meia vida longa, daí a preocupação com os efeitos do uso prolongado e do potencial para abuso (Zorzanelli et al., 2019).

É evidente que o uso de drogas combinadas prejudica e pode levar a óbito a pessoa que faz essa combinação. "O uso de diurético provoca alterações no potássio, o que causa arritmia e também desidratação, aumentando o risco de morte súbita. A combinação entre álcool e Rivotril, que é um calmante depressor do sistema nervoso central, potencializa o efeito do álcool, causando letargia, piora dos reflexos, alucinações, desatenção, confusão mental, depressão respiratória, podendo levar ao coma e à morte", explica o médico psiquiatra João Maria Corrêa Filho, do Instituto de Psiquiatria do Hospital das Clínicas, da Faculdade de Medicina da Universidade de São Paulo.

$\mathrm{O}$ estudo dos cuidados na interação farmacológica do clonazepam com o álcool faz-se necessário pelo aumento nos casos dessa interação. A preocupação com essa questão de saúde se torna mais evidente, algo que os especialistas avaliam como um mal da nossa sociedade. À medida que os problemas abrangem mais pessoas, as pesquisas tornam-se essenciais para entender e encontrar soluções no combate dessa questão.

O presente artigo é uma abordagem dos efeitos que o clonazepam em relação a depressão, insônia e ansiedade. E como é prejudicial quando usado junto ao álcool.

\section{Metodologia}

A metodologia compreende um conjunto de operações que devem ser sistematizadas e trabalhadas com consistência a partir dos seguintes procedimentos: clareza na colocação do problema; atendimento aos objetivos preestabelecidos; consiste em revisão de literatura para a construção do quadro teórico; escolha adequada dos instrumentos ou técnicas de pesquisa; definição do cronograma das atividades, coleta e análises dos dados; e conclusão com recomendações (Oliveira, 2018).

O Clonazepam é usado para tratar transtornos de pânico, crises de ansiedade, transtornos compulsivos, depressão e transtornos psicológicos. Quando um indivíduo toma Clonazepam, a droga começa a funcionar muito rapidamente. Uma vez que o Clonazepam trata os transtornos crônicos sem curá-los, com frequência as pessoas a quem esse medicamento é receitado acaba, por tomá-lo por períodos de tempo prolongados. Isso acontece porque é uma substância muito hábil na hora de relaxar os músculos rapidamente e produz um efeito hipnótico (Menezes, 2020).

$\mathrm{O}$ referido artigo teve como base uma coleta de dados sobre o tema abordado, em forma de revisão bibliográfica em trabalhos acadêmicos que possibilitaram uma melhor compreensão e expansão do assunto. O assunto é bastante difundido embora falte ainda uma melhor percepção da interação do clonazepam e álcool, procurei focar nos principais autores e os mais conceituados sites relacionados ao enfoque. A revisão narrativa foi através do site PubMed e SciELO, e de autores como: DAMACENA, LUCHO, OLIVEIRA, CARVALHO, CHELLAPPA, ANDREATINI, LOPES, DÍAZ, TORRES e revistas cientificas entre os anos de 2011 até 2021, que falam a respeito do assunto abordado, que ocorreu nos meses de setembro e outubro de 2021. Foram pesquisados 50 artigos, desses 25 compõe o artigo. 


\section{Ingestão de Álcool e Medicamentos}

A bebida alcoólica é um velho problema da sociedade, apesar de seus graves prejuízos provocados ao organismo dos indivíduos que consumem desta droga, o álcool ainda é considerado uma droga lícita, e continua sendo a droga mais consumida entre jovens que em sua maioria encontra no álcool uma função mediadora da sociabilidade e é agente de desinibição e estimulador das relações entre seus pares.

A relação entre o álcool e os medicamentos, pode ser perigosa, pois o consumo de bebidas alcoólicas pode aumentar ou diminuir o efeito do remédio, alterar o seu metabolismo, ativar a produção de substâncias tóxicas que danificam os órgãos, além de poder contribuir para a exacerbação dos efeitos secundários do medicamento, como sonolência, dor de cabeça ou vômitos. Além disso, a ingestão de álcool juntamente com medicamentos pode causar reações similares ao dissulfiram, que é um remédio usado para tratar o alcoolismo crônico, que age inibindo uma enzima que ajuda a eliminar o acetaldeído, que é um metabolito do álcool, responsável pelos sintomas da ressaca (Abreu, 2021).

O álcool é um depressor do sistema nervoso central, pois age no sistema GABA (ácido gama amino butírico, principal molécula com efeito inibitório no sistema nervoso central). Além disso, o álcool reduz níveis de serotonina no nosso cérebro à longo prazo, neurotransmissor relacionado ao humor e principal relacionado à depressão. A associação desses fatores é que gera a relação da depressão com o alcoolismo (Labinas, 2019)

$\mathrm{O}$ alcoolismo prejudica o indivíduo na sua vida escolar e na vida profissional fazendo com que ele perca o interesse em aprender e abandone os estudos e trabalho, o que acarreta na perca de confiança e autoestima e muitas vezes provocam uma forte depressão no indivíduo (Cisa, 2013)

O clonazepam que é um remédio que vai diretamente para o sistema nervoso central. O álcool inicialmente aumenta o efeito do antidepressivo, deixando a pessoa mais estimulada. Mas após passar o efeito da bebida, a pessoa se sente ainda pior, e a depressão pode aumentar por fatores como ressaca e fadiga, que a bebida pode deixar. O álcool aumenta o efeito sedativo dos antidepressivos tricíclicos, prejudicando as habilidades mentais necessárias para a condução. O consumo agudo de álcool aumenta a disponibilidade de alguns tricíclicos, potencialmente aumentando seus efeitos sedativos; O consumo crônico de álcool parece aumentar a disponibilidade de alguns tricíclicos e diminuir a disponibilidade de outros (Torres, 2019).

\section{Apresentação de Rivotril (Clonazepam)}

Clonazepam é um estimulante de GABA, um neurotransmissor inibidor neurossináptico, que tem sua atuação através do controle da abertura dos canais de cloro (Cl-), portanto, há entrada de carga negativa nas células e ocorre a diminuição da despolarização, inibindo assim as sinapses, são geralmente utilizados na forma de comprimidos pela via oral, mas há também apresentações injetáveis e líquidas (Lopes, 2019).

Clonazepam apresenta propriedades farmacológicas comuns aos benzodiazepínicos, que incluem efeitos anticonvulsivantes, sedativos, relaxantes musculares e ansiolíticos. Assim como acontece com outros benzodiazepínicos, acredita-se que esses efeitos podem ser mediados principalmente pela inibição pós-sináptica mediada pelo GABA. As pesquisas eletroencefalográficas em humanos mostraram que clonazepam suprime rapidamente muitos tipos de atividade paroxística, incluindo o aparecimento de ondas pontiagudas e descarga de ondas na ausência de convulsões (pequeno mal), ondas lentas pontiagudas, ondas pontiagudas generalizadas, espículas temporais ou de outra localização, bem como espículas e ondas irregulares (Roque químicos, 2017).

O clonazepam é prescrito por psiquiatras para o tratamento de doenças de ordem psicológicas como crises epilépticas, espasmos infantis na síndrome de West, transtornos de ansiedade, distúrbios do pânico, fobia social, transtornos do humor, transtorno bipolar, depressão, síndromes psicóticas como acatisia, síndrome das pernas inquietas, vertigens e distúrbios do equilíbrio, e no tratamento da síndrome da boca ardente. Porém o médico antes de receitar o uso do clonazepam precisa de uma 
série de exames, pois o uso desse medicamento de forma errada pode causar dependência e perder a eficácia. Além de que algumas dessas doenças citadas podem ser tratadas com outros remédios, isso irá depender da avaliação que o médico fará de cada pessoa (Beirão, 2015).

\subsection{Composição}

Princípio ativo: 5-(o-clorofenil)-1, 3-diidro-7-nitro-2H-1, 4-benzodiazepina-2-ona (clonazepam). Excipientes: Comprimidos de 0,5 mg: lactose, amido de milho, amido pregelatinizado, óxido de ferro amarelo, óxido de ferro vermelho, talco, estearato de magnésio. Comprimidos de 2,0 mg: lactose, amido pregelatinizado, estearato de magnésio, celulose microcristalina. Comprimidos sublinguais de $0,25 \mathrm{mg}$ : celulose microcristalina, manitol, amido glicolato de sódio e estearil fumarato de sódio. Gotas de 2,5 mg: sacarina sódica, ácido acético glacial, propilenoglicol, essência de pêssego (Mathias, 2020).

\subsection{Advertências}

$\mathrm{O}$ uso do clonazepam pode levar ao desenvolvimento de dependência física e psíquica. $\mathrm{O}$ risco de dependência aumenta com a dose, tratamentos prolongados e em pacientes com história de abuso de álcool ou drogas. Em caso de dependência, especialmente com doses elevadas, a descontinuação brusca do tratamento será acompanhada por sintomas de abstinência: psicoses, distúrbio de comportamento, tremor, sudorese, agitação, distúrbios do sono, dor de cabeça, dores musculares, câimbras, ansiedade extrema, tensão, cansaço, confusão, irritabilidade e convulsões que podem ser associadas à doença de base (Medley, 2020).

Em casos de dependência ou uso prolongado, quando o tratamento é interrompido bruscamente, podem ser sentidos sintomas como psicoses, distúrbios no comportamento, tremores, suor excessivo, agitação, problemas de sono, dor de cabeça, dores musculares, câimbras, ansiedade extrema, tensão, cansaço, confusão, irritabilidade ou convulsões. Durante o tratamento com Rivotril deve evitar dirigir veículos ou maquinas, pois este medicamento pode prejudicar a habilidade e a atenção. O tratamento com Rivotril nunca deve ser interrompido sem conhecimento do seu médico e os horários, as doses e duração do tratamento devem ser rigorosamente respeitados (Diaz, 2017).

\subsection{Classificação medicamentosa}

O clonazepam, nome comercial Rivotril, é um medicamento classificado como benzodiazepínico que atua como um sedativo no corpo. Este medicamento também retarda a atividade cerebral e pode causar ansiedade e pensamentos suicidas se tomado de forma inadequada. Pode desenvolver tolerância e, quando combinado com álcool, o risco dessa condição é maior. Por causa do abuso do álcool, uma pessoa perde os seus sentidos normais e pode acabar tomando mais clonazepam ao beber álcool do que quando não está bebendo normalmente. Álcool e clonazepam são depressores do sistema nervoso. Associados podem deprimir excessivamente, promover dificuldades respiratórias, aspiração de líquidos digestivos para pulmões e, dependendo da dose, coma e arritmias cardíacas (Pinheiro, 2020).

As interações medicamentosas podem ser de vários tipos a depender de qual e como o medicamento/droga vai interagir e desencadear efeitos sobre o organismo humano (Silva, 2017).

\section{Resolução no 585 de 29 de agosto de 2013}

“Com a publicação da Resolução 585/13, o farmacêutico passa a ter uma resolução para regulamentar suas atribuições clínicas, pois o documento indica os direitos e as responsabilidades desse profissional no que tange às suas áreas de atuação, principalmente relativas à saúde pública, levando em conta o cuidado à saúde, nos âmbitos individual e coletivo", fala o 
farmacêutico, professor do ICTQ - Instituto de Pesquisa e Pós-Graduação para o Mercado Farmacêutico, Alipio de Oliveira do Carmo.

Art. $7^{\circ}$ São atribuiç̃̃es clínicas do farmacêutico relativas ao cuidado à saúde, nos âmbitos individual e coletivo:

IV - Analisar a prescrição de medicamentos quanto aos aspectos legais e técnicos;

Regulamenta-se aqui a liberdade do farmacêutico em revisar a prescrição em observância às boas práticas de prescrição de cada profissional de saúde habilitado para tal.

Vale ressaltar que o farmacêutico desempenha uma função importante na monitorização da farmacoterapia prescrita e também está capacitado para avaliar aspectos relacionados aos medicamentos, garantindo, assim, a segurança do paciente. Os efeitos colaterais que ocorreram com maior frequência com Clonazepam são referentes à depressão do SNC. Algumas das reações são transitórias e desaparecem espontaneamente no decorrer do tratamento ou com a redução da dose. Elas podem ser prevenidas parcialmente pelo aumento lento da dose no início do tratamento.

\section{Interação farmacológica entre Clonazepam e Álcool - Dados fármacos}

O uso de medicamentos que causam alterações no sistema nervoso tornou-se crescente nas últimas décadas, podendo ou não produzir reações no corpo e na mente. Grande parte da população faz uso de algum psicotrópico e principalmente aqueles que são legais. O clonazepam está entre um dos mais prescritos no mundo, sendo que $10 \%$ da população teve contato com o medicamento por ano, com estimativas de que 1 a $3 \%$ já os tenha consumido regularmente por mais de um ano (Forsan, 2011; Carvalho, 2017).

Sempre que pensamos na associação de antidepressivos e álcool nos vem à cabeça que "o álcool tira o efeito do remédio", ou então que "o álcool potencializa o efeito da medicação". Há quem pense que o uso dos dois pode levar a prejuízos para o fígado ou mesmo para o cérebro. Todas essas afirmações estão certas, mas depende de qual medicamento estamos falando. Há remédios que tem seu efeito potencializado, para pior, com o álcool e outros que são inibidos pela sua ação (Labinas, 2019).

O quadro a seguir mostra dados sobre a ingestão de álcool e clonazepam. São fontes dos estudos do instituto de saúde EGAS MONIZ.

Quadro 1 - Ingestão de álcool e clonazepam.

\begin{tabular}{|l|l|l|l|l|}
\hline Álcool/Fármaco & \multicolumn{1}{|c|}{$\begin{array}{c}\text { Principal } \\
\text { Mecanismo da } \\
\text { Interação }\end{array}$} & Gravidade & Gestão & Fontes de informações \\
\hline Clonazepam & $\begin{array}{l}\text { Interação } \\
\text { Farmacodinâmica }\end{array}$ & Major & $\begin{array}{l}\text { Evitar consumo } \\
\text { de álcool }\end{array}$ & $\begin{array}{l}\text { Instituto Superior de } \\
\text { Ciências da Saúde Egas } \\
\text { Moniz }\end{array}$ \\
\hline
\end{tabular}

Fonte: Autores.

- Mecanismo de ação/ Manifestações clínicas: O Clonazepam devido aos seus efeitos depressores do SNC, quando associado à ingestão de álcool, também depressor do SNC, vai potenciar a sedação provocada pelo sinergismo de efeitos de ambas as substâncias quando administradas em conjunto (Lança, 2014).

- Relevância clínica/ Gravidade: Interação de gravidade major, bem estabelecida e clinicamente importante (Lança, 2014). 
- Gestão: Dois estudiosos têm opiniões diferentes sobre a gestão de a administração concomitante de clonazepam e álcool deve ser evitada (Truven Health Analytics, 2014); por outro, sugerem que não existe perigo de administração concomitante das duas substâncias para uma ingestão pontual e de quantidades moderadas de álcool.

\section{Resultados e Discussão}

Fazer uso de medicamentos e ingerir álcool em seguida é algo que se tornou bastante praticado, algumas pessoas fazem essa interação para aumentar o efeito da medicação sem saber o risco que está correndo. De fato, é uma preocupação e um problema de saúde que deve ser observado com mais rigor.

O consumo de clonazepam produz tolerância, e isso tem um risco maior de ocorrer quando combinado com álcool. Ao abusar do álcool, falta-nos o juízo adequado, e é provável que tomemos mais Clonazepam enquanto bebemos do que normalmente beberíamos. Isso faz um maior desenvolvimento rapidamente tolerância a medicação (Monteiro, 2021).

Medicamentos da classe dos benzodiazepínicos são responsáveis por diminuírem a atividade cerebral, casando uma leve inibição do sistema nervoso central (SNC). Leva a uma ação anticonvulsivante, sedativa, tranquilizante e relaxante muscular. Os efeitos da droga começarão a surtir efeito após 30 a 60 minutos, em média, podendo durar de 6 a 12 horas. E como já dito, esse remédio não cura nada, ele é apenas um redutor de sintomas. Sendo assim, o uso a longo prazo acaba viciando o usuário e o seu sistema nervoso. Ou seja, uma hora o Rivotril vai parar de fazer efeito (usado a longo prazo). Além disso, por ser uma droga lícita, o uso descontrolado do remédio pode causar dependência química. Existem diversos casos onde usuários do medicamento foram parar em clinicas de reabilitação (Moreira, 2016).

Tomar Clonazepam enquanto bebe álcool fará com que os batimentos cardíacos e respiratórios caiam muito rapidamente, e isso pode causar desmaios ou perda de consciência. Outros efeitos colaterais do Clonazepam incluem convulsões e problemas de memória. A combinação de clonazepam com álcool pode causar grave perda de memória e aumenta drasticamente a chance de uma convulsão. Como o clonazepam é usado para tratar doenças mentais, ele tem o potencial de piorar a doença mental quando consumido com álcool. Além disso, devido ao efeito calmante que o clonazepam e o álcool exercem sobre o corpo, as pessoas que abusam do álcool tendem a abusar do clonazepam depois de receberem esta droga. Não é incomum que pessoas viciadas em clonazepam e álcool consumam álcool em excesso, principalmente quando não estão usando a droga temporariamente (Electoral psychology, 2021).

Rivotril é um medicamento que pode causar dependência física e psicológica, pelo que, apenas deve ser utilizado de acordo com indicação médica. Alguns dos efeitos colaterais de Rivotril gotas, sublingual ou comprimidos podem incluir sonolência, lentidão de pensamento, dor de cabeça, infecções respiratórias, cansaço, gripe, depressão, vertigem, irritabilidade, insônia, falta de coordenação de movimentos e da marcha, perda do equilíbrio, náusea, sensação de cabeça leve, sinusite, falta de concentração, alterações na visão, aparência de olho esbranquiçado semelhante a catarata. Com a interação alcoólica esses efeitos ficam mais fortes podendo levar até a morte (Diaz, 2017).

\section{Considerações Finais}

Todos sabemos que qualquer medicamento precisa de receita ou acompanhamento de algum especialista para poder ser consumido, o uso abusivo e sem prescrição médica é muito comum, sendo um problema que precisa ser resolvido. As interações farmacológicas são decorrentes dessas situações, a ingestão de remédio com outra substância se torna comum, por diversos fatores, mas o principal é querer potencializar o medicamento, é o que acontece com o clonazepam e álcool. 
O presente estudo reforça os perigos que a interação do clonazepam e álcool tem sobre o organismo e o SNC de quem faz tal uso, é visível o quanto a pessoa que consome, seja por prazer ou dependência, se torna mais vulnerável a certas doenças, podendo levar até a morte.

Contudo, o uso de substâncias que potencializam outra é um risco eminente e deve-se ter um controle mais rigoroso sobre tal questão. A interação farmacológica do clonazepam com álcool é algo que acontece com mais frequência do que imaginamos e, é um assunto que deve ser mais analisado e estudado.

Como sugestão para trabalhos futuros, pesquisas com pessoas com idade acima de 35 anos podem ser melhores exploradas, visto que é nessa faixa etária que os problemas com ansiedade e síndrome do pânico são mais recorrentes.

\section{Referências}

Clonazepam medley. (2020). clonazepam Medley - Bula Completa do Medicamento - PR Vade mécum (prvademecum.com)

Damacena, G. N. et al. (2016). Consumo abusivo de álcool e envolvimento em acidentes de trânsito na população brasileira, 2013. Ciênc. saúde coletiva. 21(12). http://dx.doi.org/10.1590/1413-812320152112.25692015

Deus, P. O. (2020). Avaliação do uso de clonazepam pela população vinculada a uma unidade do Programa Saúde da Família em Teófilo Otoni. https://www.bibliomed.com.br/lib/showdoc.cfm?LibDocID=16211.

Diaz, T. T. (2017). Rivotril bula - Para que serve e efeitos colaterais (bulario.com)

DRUGDEX® System. MICROMEDEX® Truven Health Analytics. The Healthcare Business of Thomson Reuters. (2014). http://www.micromedexsolutions.com/home/dispatch.

Efeitos colaterais do rivotril. (2021). Efeitos Colaterais do Rivotril (Clonazepam) (e-tudo.net)

Leonardi, J. N. et al. (2017). Benzodiazepinicos e seus efeitos no sistema nervoso central. Revista Saúde em Foco - Edição n ${ }^{\circ} 9$ - Ano: 2017

Leite, P. (2019). Antidepressivos e Álcool - Antidepressivos e Álcool - Efeitos e Riscos - MundoBoaForma.

Labinas, L.G.O. (2019). Antidepressivos e bebidas alcoólicas: Pode misturar? Veja o que acontece. Antidepressivos e bebidas alcoólicas: Pode misturar? Veja o que acontece | Minha Vida.

Lopes, F. F. de P. (2019). Microsoft PowerPoint - Direito a Informação (anvisa.gov.br).

Mathias, F. T. (2020). Bula do Clonazepam: Clonazepam: Bula original, extraída manualmente da Anvisa | CR (consultaremedios.com.br).

Medic. (2021). Clonazepam Bula: Entenda para que serve e como tomar. 4medic.com.br.

Moreira, D. (2016). Entenda porque o rivotril é tão perigoso para a saúde. Entenda porque o Rivotril é tão perigoso para a saúde - Mundo Inverso.

Pinheiro, P. (2020). Clonazepam (Rivotril): para que serve e efeitos. Clonazepam (Rivotril): para que serve e efeitos - MD.Saúde (mdsaude.com).

Pires, M. T. B. (2019). Bibliomed - Clonazepam - artigo médico de revisão para pesquisa e consulta.

RESOLUÇÃO N 585 de 29 de agosto de 2013. Ementa: Regulamenta as atribuições clínicas do farmacêutico e dá outras providências. (2013). Relatório da comissão designada para estudar a manutenção da terminologia "Bioquímico" ao farmacêutico, após a implantação das diretrizes curriculares. (cff.org.br).

Rivotril faz mal? Efeitos colaterais e cuidados. (2020). MundoBoaForma. Rivotril Faz Mal? Efeitos Colaterais e Cuidados - MundoBoaForma

Se eu tomar clonazepam, posso beber álcool? (2021). pt.sainte-anastasie.org.

Se eu tomar clonazepam, posso beber álcool? - Vícios. (2021). (electoralpsychology.com)

Silva, M. A. A. (2014). O impacto do alcoolismo na vida social e familiar do indivíduo: A intervenção do profissional da saúde de forma efetiva no tratamento. Sr (ufmg.br)

Silva, M. H.; Teixeira, D. A. \& Costa; R. C. A. (2020). O uso indiscriminado de clonazepam e a importância da assistência de enfermagem. ISSN: 2674-8584 V.1 - N.1 - 2020

Torres, P. (2019). Medicamentos e bebidas alcoólicas, uma relação perigosa. Medicamentos e bebidas alcoólicas, uma relação perigosa - Artrite Reumatoide.

Zorzanelli, R. T. et al. (2019). Consumo do benzodiazepínico clonazepam (Rivotrilß) no estado do Rio de Janeiro, Brasil, 2009-2013: estudo ecológico. https://doi.org/10.1590/1413-81232018248.23232017 